\title{
In Situ Synthesis of Nanosized NiO Encapsulated in Graphene as High-performance Supercapacitor Cathode
}

\author{
Danfeng Qiu ${ }^{1, *}$, Xiao Ma ${ }^{1}$, Jingdong Zhang ${ }^{1}$, Zixia Lin $^{2}$ and Bin Zhao ${ }^{2}$ \\ ${ }^{1}$ Key Laboratory of Radar Imaging and Microwave Photonics (Nanjing Univ. Aeronaut. Astronaut.), \\ Ministry of Education, College of Electronic and Information Engineering, Nanjing University of Aero \\ nautics and Astronautics, Nanjing, 210016, China \\ ${ }^{2}$ National Laboratory of Microstructures and School of Electronic Science and Engineering, Nanjing \\ University, Nanjing, China \\ *E-mail: dfqiu@nuaa.edu.cn
}

doi: $10.20964 / 2018.09 .35$

Received: 13 May 2018 / Accepted: 2 July 2018 / Published: 5 August 2018

\begin{abstract}
$\mathrm{NiO}$ nanoparticles embedded in graphene nanosheets (GNS) were successfully prepared by coprecipitation and pyrolysis beforehand. The three-dimensional GNS matrix with interconnected pore network structure can be filled with electrolyte ions serving as a double ion buffer pool during charge and discharge. High conductivity graphene, as a three-dimensional network, can be used to support the fast transmission of charge inside the $\mathrm{NiO}$ electrode during charging and discharging processes and meanwhile increase the performance rate. Hence, the NiO/GNS nanocomposites exhibit specific capacitance value of $1125.8 \mathrm{~F} \mathrm{~g}^{-1}$ at a current density of $2 \mathrm{~A} \mathrm{~g} \mathrm{~g}^{-1}$ in $2 \mathrm{M} \mathrm{KOH}$ solution. This composite exhibits a good cyclic stability, with a small loss of 5.2\% of maximum capacitance over a consecutive 2000 cycles, indicating that the prepared NiO/GNS nanocomposites serve as potential and promising candidates for supercapacitor electrodes.
\end{abstract}

Keywords: Graphene; Nickel oxide; Nanocomposites; Energy storage and conversion; Supercapacitors

\section{$\underline{\text { FULL TEXT }}$}

(C) 2018 The Authors. Published by ESG (www.electrochemsci.org). This article is an open access article distributed under the terms and conditions of the Creative Commons Attribution license (http://creativecommons.org/licenses/by/4.0/). 